\title{
Sex differences in the association between socioeconomic status and diabetes prevalence and incidence in China: cross-sectional and prospective studies of 0.5 million adults
}

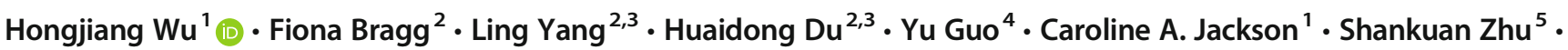 \\ Canqing $\mathrm{Yu}^{6} \cdot$ Andrea O. Y. Luk $^{7}$. Juliana C. N. Chan ${ }^{7}$. Danijela Gasevic ${ }^{1,8} \cdot$ Liming Li $^{4,6} \cdot$ Zhengming Chen $^{2}$. \\ Sarah H. Wild ${ }^{1}$
}

Received: 12 February 2019 / Accepted: 10 April 2019 / Published online: 31 May 2019

(C) The Author(s) 2019

\begin{abstract}
Aims/hypothesis China has undergone rapid socioeconomic transition accompanied by lifestyle changes that are expected to have a profound impact on the health of its population. However, there is limited evidence from large nationwide studies about the relevance of socioeconomic status (SES) to risk of diabetes. We describe the associations of two key measures of SES with prevalent and incident diabetes in Chinese men and women.

Methods The China Kadoorie Biobank study included 0.5 million adults aged 30-79 years recruited from ten diverse areas in China during 2004-2008. SES was assessed using the highest educational level attained and annual household income. Prevalent diabetes was identified from self-report and plasma glucose measurements. Incident diabetes was identified from linkage to disease and death registries and national health insurance claim databases. We estimated adjusted ORs and HRs for prevalent and incident diabetes associated with SES using logistic and Cox regression models, respectively.

Results At baseline, 30,066 (5.9\%) participants had previously diagnosed (3.1\%) or screen-detected (2.8\%) diabetes among 510,219 participants included for cross-sectional analyses. There were 480,153 people without prevalent diabetes at baseline, of whom 9544 (2.0\%) had new-onset diabetes during follow-up (median 7 years). Adjusted ORs (95\% CIs) for prevalent diabetes, comparing highest vs lowest educational level, were $1.21(1.09,1.35)$ in men and $0.69(0.63,0.76)$ in women; for incident diabetes, the corresponding HRs were $1.27(1.07,1.51)$ and $0.80(0.67,0.95)$, respectively. For household income, the adjusted ORs for prevalent diabetes, comparing highest vs lowest categories, were $1.45(1.34,1.56)$ in men and $1.26(1.19$, 1.34$)$ in women; for incident diabetes, the HRs were $1.36(1.19,1.55)$ and $1.06(0.95,1.17)$, respectively.

Conclusions/interpretation Among Chinese adults, the associations between education and diabetes prevalence and incidence differed qualitatively between men and women, whereas higher household income was positively associated with diabetes prevalence and incidence in both sexes, with a stronger relationship in men than in women.
\end{abstract}

Keywords Diabetes $\cdot$ Educational level $\cdot$ Health inequality $\cdot$ Household income $\cdot$ Socioeconomic status

Electronic supplementary material The online version of this article (https://doi.org/10.1007/s00125-019-4896-z) contains peer-reviewed but unedited supplementary material, which is available to authorised users.

Sarah H. Wild

sarah.wild@ed.ac.uk

1 Usher Institute of Population Health Sciences and Informatics, University of Edinburgh, Teviot Place, Edinburgh EH8 9AG, UK

2 Nuffield Department of Population Health, University of Oxford, Oxford, UK

3 Medical Research Council Population Health Research Unit at the University of Oxford, Oxford, UK
4 Chinese Academy of Medical Sciences, Beijing, China

5 School of Public Health, Zhejiang University, Hangzhou, China

6 Department of Epidemiology and Biostatistics, School of Public Health, Peking University Health Science Center, Beijing, China

7 Department of Medicine and Therapeutics, The Chinese University of Hong Kong, Prince of Wales Hospital, Shatin, Hong Kong, China

8 School of Public Health and Preventive Medicine, Monash University, Melbourne, VIC, Australia 


\section{Research in context}

\section{What is already known about this subject?}

- In developed countries, type 2 diabetes is more prevalent among groups of lower compared with higher socioeconomic status (SES), whereas the opposite pattern has been found in developing countries

- Previous studies have reported inconsistent associations between SES and prevalence of type 2 diabetes in mainland China

- A consistent inverse association between SES and prevalence of type 2 diabetes in Hong Kong and Taiwan has been reported

\section{What is the key question?}

- What is the association between SES and prevalence and incidence of type 2 diabetes in China?

\section{What are the new findings?}

- Household income was positively associated with diabetes prevalence and incidence in both men and women, with a weaker association in women than in men

- Educational level was positively associated with prevalent and incident diabetes in men. However, there was an inverse association between educational level and diabetes prevalence and incidence in women

- $\quad$ BMl appeared to be an important mediating variable between SES and diabetes prevalence and incidence in Chinese men and women

\section{How might this impact on clinical practice in the foreseeable future?}

- The findings of this study provide information that will be useful in identifying priority groups for diabetes prevention and in future evaluation of the effect of policies to reduce inequalities in China

$\begin{array}{ll}\text { Abbreviations } \\ \text { CKB } & \text { China Kadoorie Biobank } \\ \text { LMIC } & \text { Low- and middle-income countries } \\ \text { MET } & \text { Metabolic equivalent task } \\ \text { NCD } & \text { Non-communicable disease } \\ \text { SES } & \text { Socioeconomic status } \\ \text { TIA } & \text { Transient ischaemic attack }\end{array}$

\section{Introduction}

The prevalence of diabetes in China has increased markedly in the past few decades. The proportion of Chinese adults estimated to have diabetes was $0.9 \%$ in $1980,2.5 \%$ in 1994 and $10.9 \%$ in 2013 [1-3]. The increase is thought to be the most rapid worldwide, and it is related to China's recent rapid economic development and urbanisation, which are contributing to socioeconomic and epidemiological transition [4].

Evidence from developed countries that have completed the epidemiological transition shows that non-communicable diseases (NCDs) are initially more common in population subgroups of high socioeconomic status (SES) and then, with increasing development, become more common in lower SES groups [5]. However, the evidence from low- and middleincome countries (LMIC) is limited [6]. Previous studies have reported inconsistent associations between SES and diabetes prevalence in mainland China, and findings were not obviously influenced by study year or the level of economic development of the study area [7, 8]. However, in Hong Kong and Taiwan, where economic development and epidemiological transition are at a more advanced stage than in mainland China, an inverse association between SES and diabetes prevalence has been described [7]. Reliable assessment of the association between SES and diabetes in different parts of China is needed to plan and evaluate health services and diabetes prevention strategies. In order to examine the recent socioeconomic pattern of diabetes risk in China, we describe the associations between SES and both prevalent and incident diabetes, using data from the China Kadoorie Biobank (CKB), a large prospective cohort study of about 0.5 million Chinese adults.

\section{Methods}

Study population Detailed information about the study design, survey methods and population of the CKB has been reported previously [9]. Briefly, the baseline survey took place between June 2004 and July 2008 in ten geographically defined areas (five urban and five rural) of China. The areas were selected according to local disease patterns, exposure to certain risk factors, population stability, levels of SES, quality of 
death and disease registries, local commitment and capacity. Overall, 512,891 adults aged 30-79 years were enrolled, with a response rate of about $30 \%$.

Ethical approval for this study was obtained from the University of Oxford, the Chinese Centre for Disease Control and Prevention and the local Centres for Disease Control and Prevention in the ten study regions. All participants provided written informed consent.

Assessment of socioeconomic status We assessed participants' SES status in two ways: (1) the highest level of school education an individual attained (no formal school, primary school, middle or high school, and college or above); and (2) total household income in the previous year $(<10,000$, 10,000-19,999, 20,000-34,999, and $\geq 35,000$ Chinese yuan).

Assessment of covariates We obtained covariates from the baseline questionnaire, including demographic characteristics (age, sex, urban and rural residence, and study regions), health-related behaviours (regular active and passive smoking, regular consumption of alcohol, fresh fruit, fresh vegetables and fish), personal and family medical history (history of CHD, stroke or transient ischaemic attack [TIA], cancer and diabetes). Regular active smoking was defined as current smoking on most days or more. Regular passive smoking was defined as exposure to other people's tobacco smoke for 4 or more days per week. Regular consumption of alcohol was defined as drinking alcohol monthly or more frequently. Regular consumption of fresh fruit, vegetables and fish was defined as consumption of these foods on four or more days per week. A range of physical measurements were undertaken by trained technicians using a standard protocol and calibrated instruments, including BMI, waist and hip circumference, fat percentage and systolic and diastolic BP. Physical activity was estimated by summing the metabolic equivalent task (MET) h per day spent on work, commuting, housework and non-sedentary recreational activities [10]. Economic-geographic areas of China were categorised into four groups according to the National Bureau of Statistics of China and ranked from high to low per capita disposable income of households as Eastern, Northeastern, Central and Western, which reflected different levels of economic development in China [11]. For BMI, we used cut-off points for Chinese populations to define overweight $\left(\geq 24 \mathrm{~kg} / \mathrm{m}^{2}\right.$ and $<28 \mathrm{~kg} / \mathrm{m}^{2}$ ) and obesity $\left(\geq 28 \mathrm{~kg} / \mathrm{m}^{2}\right)$ [12].

\section{Baseline prevalent diabetes, follow-up and ascertainment of} incident diabetes A $10 \mathrm{ml}$ non-fasting blood sample was collected from study participants at baseline and fasting time was recorded. Plasma glucose levels were tested on-site using the SureStep Plus meter (LifeScan, Shanghai, China). Participants with plasma glucose levels from $7.8 \mathrm{mmol} / 1$ to less than $11.1 \mathrm{mmol} / \mathrm{l}$ were invited to return for a fasting plasma glucose test on the following day. Participants were asked at baseline:
'Has a doctor ever told you that you had diabetes?', with those reporting 'yes' defined as having self-reported previously diagnosed diabetes. Screen-detected diabetes was defined among participants without self-reported diabetes on the basis of any of: (1) random plasma glucose level $\geq 7.0 \mathrm{mmol} / \mathrm{l}$ and a fasting time $\geq 8 \mathrm{~h}$; (2) random plasma glucose level $\geq 11.1 \mathrm{mmol} / \mathrm{l}$ and a fasting time $<8 \mathrm{~h}$; and (3) fasting plasma glucose level $\geq 7.0 \mathrm{mmol} / \mathrm{l}$. Prevalent diabetes includes both self-reported previously diagnosed diabetes and screen-detected diabetes.

The vital status of participants was obtained periodically from local death registries based at China's Disease Surveillance Points system, checked annually against local residential records and health insurance records and confirmed with street committees or village administrators. Incident diabetes was identified from linkage to disease and death registries and national health insurance databases, collecting details of diagnoses resulting in, or during, any hospital admission via individuals' unique national ID. We defined incident diabetes using the E10-E14 codes from the Tenth Revision of the International Classification of Diseases (http://apps.who.int/ classifications/icd10/browse/2016/en).

Statistical analysis We excluded $2672(0.5 \%)$ participants with missing, implausible or extreme values for all variables, leaving 510,219 participants for cross-sectional analyses of prevalent diabetes (electronic supplementary material [ESM] Fig. 1). We excluded 30,066 (5.9\%) participants with previously diagnosed diabetes $(3.1 \%)$ or screen-detected diabetes $(2.8 \%)$ at baseline, leaving 480,153 participants for prospective analyses of incident diabetes. All analyses were stratified by sex. Logistic regression was used to estimate ORs and 95\% CIs for the association between SES and baseline prevalent diabetes. For educational level, we adjusted for confounding variables including age at baseline (continuous), ten study regions, family history of diabetes, and household income in model 1 . To further assess potential mediating variables for the association of SES with diabetes, we added BMI as a continuous variable to model 1 to create model 2 . In model 3 , we added other potential mediating variables, including waist circumference, fat percentage, physical activity, regular alcohol consumption, regular active smoking, regular passive smoking, consumption of fresh fruit, fresh vegetables and fish, history of CHD, stroke or TIA and cancer, systolic BP and diastolic BP. The potential mediating variables were selected based on prior knowledge of underlying mechanisms linking SES and diabetes [13, 14]. For household income, we built similar models but further adjusted for educational level and household size in all models. There was no evidence of serious multicollinearity in any model based on values of variance inflation factors.

To estimate incidence, we calculated person-years from baseline until the date of first record of incident diabetes, death, loss to follow-up or 31 December 2013, whichever came first. We used stratified Cox regression models to 
estimate the HRs and $95 \%$ CIs for the association between SES and incident diabetes, with various models constructed in similar ways to those used for analysing prevalent diabetes. We checked the Cox proportional hazards assumption using Schoenfeld residuals and found the assumption was not violated for any model. We performed likelihood ratio tests to investigate potential interactions between SES and variables of interest, by comparing models with and without interaction terms, and investigated potential linear trend effects of SES on incident diabetes by comparing models including and excluding a linear effect term.

We performed further analyses to investigate the impact of a composite measure of educational level and household income on incident diabetes. We categorised participants into four groups: (1) low educational level and low income; (2) low educational level and high income; (3) high educational level and low income; and (4) high educational level and high income. Low educational level was defined as having education at primary school level or below; low household income was defined as having annual household income $<20,000$ Chinese yuan. Additional sensitivity analyses were done after excluding individuals with baseline CHD, stroke or TIA, or cancer, to reduce potential reverse causality. R software (version 3.3.3; www.R-project.org, Vienna, Austria) was used to perform the analyses.

\section{Results}

Characteristics of participants and patterns of covariates with SES Among 510,219 participants included for cross-sectional analyses, $59.0 \%$ were women and $44.1 \%$ were from urban areas (Table 1). The mean (SD) age was 52.3 (10.9) years for men and 50.9 (10.5) years for women. Higher proportions of men than women received college-level education or above $(7.9 \%$ vs $4.5 \%)$ and were in the highest household income group (20.3\% vs $16.5 \%$ ), and lower proportions of men than women received no formal education $(8.9 \%$ vs $25.2 \%)$ and were in the lowest household income group (26.0\% vs $29.7 \%$ ). Men with higher educational level and household income were more likely to be overweight or obese and had higher BMI, waist circumference and percentage fat than those in lower SES groups (ESM Tables 1 and 2). The absolute differences in these covariates by SES in women were very small, with values slightly higher in both the middle educational level and the middle household income groups compared with those at either extreme (ESM Tables 1 and 2).

Associations between SES and prevalent diabetes Agestandardised diabetes prevalence increased with higher educational level and household income in men, but the patterns were less clear in women (Table 2). After adjustment for age, study region, family history of diabetes and household income, there was a positive association between educational level and prevalent diabetes in men and an inverse association in women $\left(p_{\text {interaction }}<0.0001\right.$ between sex) (Fig. 1). The ORs (95\% CIs) derived from model 1, comparing highest vs lowest educational level, were $1.21(1.09,1.35)$ in men and 0.69 $(0.63,0.76)$ in women. There was a positive association between household income and prevalent diabetes in both men and women, with the association more pronounced in men than in women ( $p_{\text {interaction }}<0.0001$ between sex) (Fig. 1). The ORs (95\% CIs), comparing highest vs lowest household income category from model 1 , were $1.45(1.34,1.56)$ in men and $1.26(1.19,1.34)$ in women. These associations were attenuated after additional adjustment for BMI but remained statistically significant, changing slightly after further adjustment for other potential mediating variables (Fig. 1, ESM Tables 3 and 4).

Associations between SES and incident diabetes The characteristics of participants without diabetes at baseline ( $n=480,153$ ) by SES were similar to those of people included for cross-sectional analyses (ESM Tables 5-7). During a median of 7 years (3.4 million person-years total) of follow-up, $2032(0.4 \%)$ participants were lost to follow-up and 22,103 (4.6\%) participants died. In total, 9544 new cases of diabetes were identified, 5937 among women (Table 3). Agestandardised diabetes incidence increased with higher household income in both men and women. A U-shaped association was observed between educational level and diabetes incidence because the lowest incidence of diabetes occurred in both men and women who had received a maximum of middle or high school education (Table 3). The associations of SES with incident diabetes were similar to those with prevalent diabetes. In model 1, educational level was positively associated with incident diabetes in men, but an inverse association was found in women $\left(p_{\text {interaction }}<0.0001\right.$ between sex) (Fig. 2). The adjusted HRs (95\% CIs), comparing highest vs lowest educational level, were $1.27(1.07,1.51)$ in men and $0.80(0.67,0.95)$ in women. Household income was also positively associated with incident diabetes in men, but there was a nonsignificant positive association between household income and incident diabetes in women ( $p_{\text {interaction }}<$ 0.0001 between sexes) (Fig. 2). The adjusted HRs (95\% CIs), comparing highest vs lowest household income category, were $1.36(1.19,1.55)$ in men and $1.06(0.95,1.17)$ in women. There was no evidence of a departure from linear trend for any analysis ( $p \geq 0.05$ ), except for educational level and incident diabetes in women $(p=0.047)$. After additional adjustment for BMI, the associations between SES and incident diabetes were markedly attenuated and no longer statistically significant at the 5\% level for any comparison between the highest and lowest SES groups (Fig. 2, ESM Tables 8 and 9). Further adjustment 
Table 1 Sex-specific characteristics of CKB participants included in cross-sectional analyses

\begin{tabular}{|c|c|c|c|}
\hline Variable & Men $(n=209,352)$ & Women $(n=300,867)$ & All $(n=510,219)$ \\
\hline Age, years & $52.3(10.9)$ & $50.9(10.5)$ & $51.5(10.7)$ \\
\hline Urban residence, $n(\%)$ & $90,936(43.4)$ & $133,913(44.5)$ & $224,849(44.1)$ \\
\hline \multicolumn{4}{|l|}{ Geographic area, $n(\%)$} \\
\hline Eastern & $72,531(34.6)$ & $102,692(34.1)$ & $175,233(34.3)$ \\
\hline Northeastern & $23,170(11.1)$ & $34,164(11.4)$ & $57,334(11.2)$ \\
\hline Central & $54,038(25.8)$ & $68,757(22.9)$ & $122,795(24.1)$ \\
\hline Western & $59,613(28.5)$ & $95,254(31.7)$ & $154,867(30.4)$ \\
\hline \multicolumn{4}{|l|}{ Educational level, $n(\%)$} \\
\hline No formal school & $18,529(8.9)$ & $75,834(25.2)$ & $94,363(18.5)$ \\
\hline Primary school & $69,769(33.3)$ & $94,571(31.4)$ & $164,340(32.2)$ \\
\hline Middle or high school & $104,569(49.9)$ & $117,037(38.9)$ & $221,606(43.4)$ \\
\hline College or above & $16,485(7.9)$ & $13,425(4.5)$ & $29,910(5.9)$ \\
\hline \multicolumn{4}{|l|}{ Household income (yuan/year), $n(\%)$} \\
\hline$<10,000$ & $54,386(26.0)$ & 89,437 (29.7) & $143,823(28.2)$ \\
\hline $10,000-19,999$ & $59,335(28.3)$ & $88,959(29.6)$ & $148,294(29.1)$ \\
\hline $20,000-34,999$ & $53,229(25.4)$ & $72,911(24.2)$ & $126,140(24.7)$ \\
\hline$\geq 35,000$ & $42,402(20.3)$ & $49,560(16.5)$ & $91,962(18.0)$ \\
\hline Regular alcohol consumption, $n(\%)$ & $93,142(44.5)$ & $11,738(3.9)$ & $104,880(20.6)$ \\
\hline \multicolumn{4}{|l|}{ Regular smoking, $n(\%)$} \\
\hline Active & $127,862(61.1)$ & $7103(2.4)$ & $134,965(26.5)$ \\
\hline Passive & $116,683(55.7)$ & $156,950(52.2)$ & $273,633(53.6)$ \\
\hline \multicolumn{4}{|l|}{ Regular consumption of foods, $n(\%)$} \\
\hline Fresh fruit & $48,237(23.0)$ & $95,693(31.8)$ & $143,930(28.2)$ \\
\hline Fresh vegetables & $205,726(98.3)$ & $295,846(98.3)$ & $501,572(98.3)$ \\
\hline Fish & $19,820(9.5)$ & $25,499(8.5)$ & $45,319(8.9)$ \\
\hline \multicolumn{4}{|l|}{ Prior diseases, $n(\%)$} \\
\hline CHD & $5683(2.7)$ & $9654(3.2)$ & $15,337(3.0)$ \\
\hline Stroke or TIA & $4873(2.3)$ & $3926(1.3)$ & $8799(1.7)$ \\
\hline Cancer & $963(0.46)$ & $1599(0.53)$ & $2562(0.50)$ \\
\hline Family history of diabetes, $n(\%)$ & $9894(4.7)$ & $15,130(5.0)$ & $25,024(4.9)$ \\
\hline \multicolumn{4}{|l|}{ BMI $\left(\mathrm{kg} / \mathrm{m}^{2}\right)$} \\
\hline Mean & $23.4(3.2)$ & $23.8(3.4)$ & $23.7(3.4)$ \\
\hline$<24$ (normal), $n(\%)$ & $122,143(58.3)$ & $164,643(54.7)$ & $286,786(56.2)$ \\
\hline 24-28 (overweight), $n(\%)$ & $68,628(32.8)$ & $100,927(33.5)$ & $169,555(33.2)$ \\
\hline$\geq 28$ (obese), $n(\%)$ & $18,581(8.9)$ & $35,297(11.7)$ & $53,878(10.6)$ \\
\hline Physical activity, MET-h/day & $22.0(15.3)$ & $20.4(12.8)$ & $21.1(13.9)$ \\
\hline Systolic BP, mmHg & $132.8(20.0)$ & $129.8(21.9)$ & $131.1(21.2)$ \\
\hline Diastolic BP, mmHg & $79.2(11.3)$ & $76.8(10.9)$ & $77.8(11.1)$ \\
\hline Waist circumference, $\mathrm{cm}$ & $82.0(9.7)$ & $79.1(9.5)$ & $80.3(9.7)$ \\
\hline Fat, \% & $22.0(6.2)$ & $32.1(7.1)$ & $30.0(8.4)$ \\
\hline
\end{tabular}

Values are shown as $n(\%)$ or mean (SD) for other potential mediating factors had little effect on the results (ESM Tables 8 and 9).

The association between educational level and incident diabetes did not appear to be modified by age, urban or rural residence, economic-geographic areas or household income in men or women (ESM Fig. 2). However, the positive association of household income with incident diabetes was more pronounced in urban areas than in rural areas in men $\left(p_{\text {interaction }}=0.035\right)$ but not in women $\left(p_{\text {interaction }}=0.99\right)$ and was more pronounced in less economically developed geographic areas (Northeastern, Central and Western) than in more developed areas (Eastern) in both sexes $\left(p_{\text {interaction }}=0.017\right.$ in men and $p_{\text {interaction }}=$ 0.010 in women) (ESM Fig. 3). Absolute risk of incident diabetes was lowest in men with low household income regardless of educational level and highest in women with low educational level and high household income (ESM Table 10). The relative risk of developing diabetes compared with the groups with low educational level and low household income was highest in men who had both high educational level and high household income and in women who had low educational level but high household income (ESM Table 10). Excluding people with a history of any of CHD, stroke, TIA or cancer at baseline from the study population had no effect on the associations of SES with prevalent or incident diabetes (ESM Tables 11 and 12).

\section{Discussion}

This large nationwide prospective study in about 0.5 million Chinese adults showed that the associations between SES and diabetes prevalence and incidence differ between men and women. Among men, both educational level and household income were positively associated with risk of diabetes. Among women, there was an inverse association between educational level and risk of diabetes, whereas for household 
Table 2 Sex-specific diabetes prevalence among CKB participants included in cross-sectional analyses according to educational level and household income

\begin{tabular}{|c|c|c|c|c|}
\hline \multirow[t]{2}{*}{ Socioeconomic status } & \multicolumn{2}{|l|}{ Men $(n=209,352)$} & \multicolumn{2}{|c|}{ Women $(n=300,867)$} \\
\hline & $\begin{array}{l}\text { No. of participants } \\
\text { with prevalent } \\
\text { diabetes }\end{array}$ & $\begin{array}{l}\text { Standardised } \\
\text { prevalence rate, }{ }^{\text {a }} \% \\
(95 \% \text { CI })\end{array}$ & $\begin{array}{l}\text { No. of participants } \\
\text { with prevalent } \\
\text { diabetes }\end{array}$ & $\begin{array}{l}\text { Standardised prevalence } \\
\text { rate, }{ }^{\text {a }} \%(95 \% \mathrm{CI})\end{array}$ \\
\hline All & 11,616 & $5.55(5.45,5.65)^{\mathrm{b}}$ & 18,450 & $6.13(6.04,6.22)^{\mathrm{b}}$ \\
\hline \multicolumn{5}{|l|}{ Educational level } \\
\hline No formal school & 863 & $3.55(3.47,3.64)$ & 5523 & $5.47(5.30,5.64)$ \\
\hline Primary school & 3444 & $4.24(4.15,4.33)$ & 6230 & $6.17(6.01,6.33)$ \\
\hline Middle and high school & 5950 & $6.91(6.81,7.02)$ & 6033 & $7.45(7.24,7.67)$ \\
\hline College or above & 1359 & $9.03(8.91,9.15)$ & 664 & $6.97(6.43,7.54)$ \\
\hline \multicolumn{5}{|l|}{ Household income, yuan/year } \\
\hline$<10,000$ & 2232 & $3.86(3.77,3.95)$ & 5037 & $5.27(5.19,5.34)$ \\
\hline $10,000-19,999$ & 3400 & $5.75(5.55,5.94)$ & 5802 & $6.65(6.48,6.82)$ \\
\hline $20,000-34,999$ & 3238 & $6.31(6.09,6.53)$ & 4544 & $6.61(6.42,6.81)$ \\
\hline$\geq 35,000$ & 2746 & $6.94(6.68,7.21)$ & 3067 & $6.49(6.26,6.73)$ \\
\hline
\end{tabular}

${ }^{a} 5$ year age group standardised to the whole CKB population

${ }^{\mathrm{b}}$ Crude prevalence

No., number

income the positive associations with diabetes prevalence and incidence were weaker than in men. The associations of

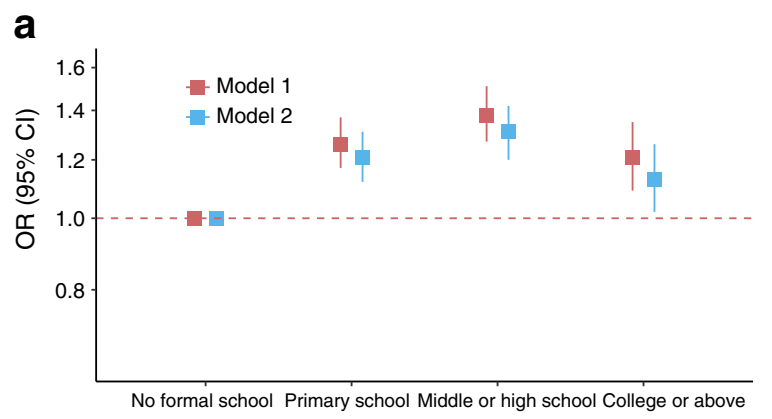

Educational level

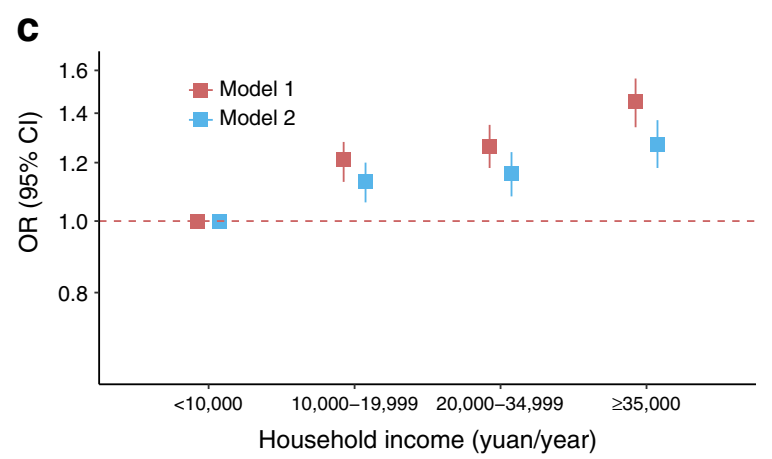

Fig. 1 Adjusted ORs and 95\% CIs for prevalent diabetes associated with educational level $(\mathbf{a}, \mathbf{b})$ and household income $(\mathbf{c}, \mathbf{d})$ in men $(\mathbf{a}, \mathbf{c})$ and women $(\mathbf{b}, \mathbf{d})$, plotted on a logarithmic scale. For educational level, model 1 was adjusted for age at baseline (continuous), study region, family history of diabetes and household income. Model 2 was further adjusted educational level and household income with diabetes appeared to be partly mediated by BMI.
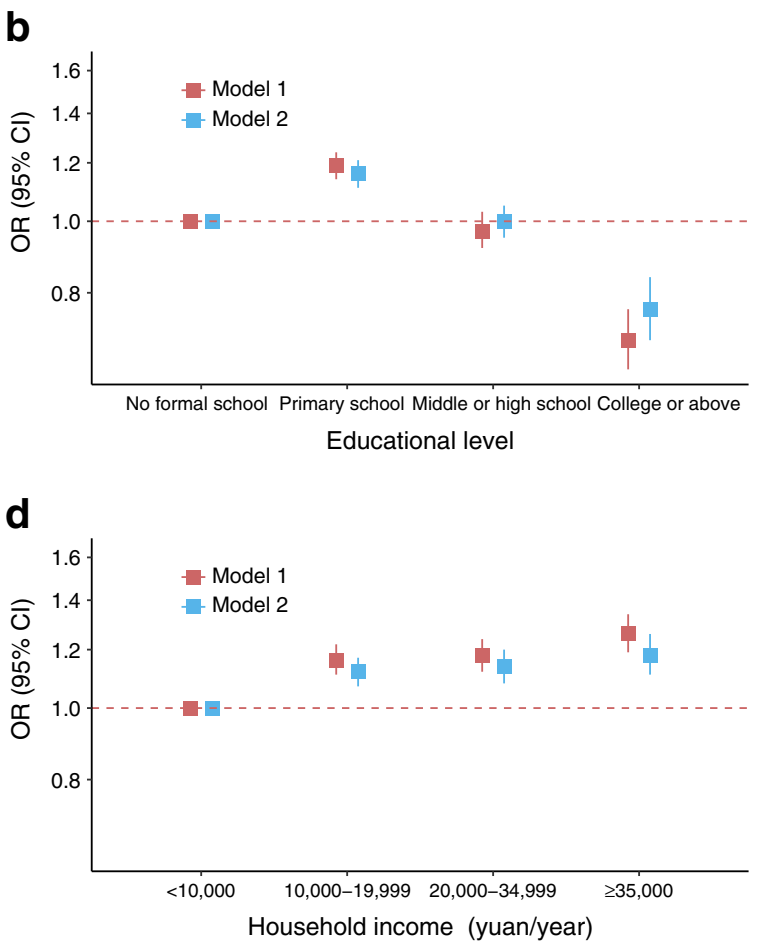

for BMI based on model 1. For household income, model 1 was adjusted for age at baseline (continuous), study region, family history of diabetes, educational level and household size. Model 2 was further adjusted for BMI based on model 1. See ESM Tables 3 and 4 for data 
Table 3 Sex-specific diabetes incidence among CKB participants included in prospective analyses according to educational level and household income

\begin{tabular}{|c|c|c|c|c|}
\hline \multirow[t]{2}{*}{ Socioeconomic status } & \multicolumn{2}{|l|}{$\operatorname{Men}(n=197,736)$} & \multicolumn{2}{|l|}{ Women $(282,417)$} \\
\hline & $\begin{array}{l}\text { No. of participants } \\
\text { with incident diabetes }\end{array}$ & $\begin{array}{l}\text { Standardised incidence } \\
\text { rate }^{\mathrm{a}} \text { (no./1000 person-years) }\end{array}$ & $\begin{array}{l}\text { No. of participants } \\
\text { with incident diabetes }\end{array}$ & $\begin{array}{l}\text { Standardised incidence } \\
\text { rate }^{\mathrm{a}} \text { (no./1000 person-years) }\end{array}$ \\
\hline All & 3607 & $2.59(2.51,2.68)^{\mathrm{b}}$ & 5937 & $2.93(2.86,3.01)^{\mathrm{b}}$ \\
\hline \multicolumn{5}{|l|}{ Highest educational level } \\
\hline No formal school & 480 & $3.38(2.98,3.85)$ & 2330 & $3.80(3.62,3.99)$ \\
\hline Primary school & 1342 & $2.65(2.49,2.81)$ & 1945 & $2.96(2.83,3.10)$ \\
\hline Middle or high school & 1469 & $2.37(2.25,2.51)$ & 1485 & $2.29(2.16,2.43)$ \\
\hline College or above & 316 & $3.24(2.88,3.64)$ & 177 & $2.51(2.13,2.94)$ \\
\hline \multicolumn{5}{|l|}{ Household income, yuan/year } \\
\hline$<10,000$ & 747 & $1.86(1.73,2.00)$ & 1522 & $2.36(2.23,2.48)$ \\
\hline $10,000-19,999$ & 835 & $2.11(1.97,2.25)$ & 1536 & $2.59(2.46,2.72)$ \\
\hline $20,000-34,999$ & 1003 & $2.95(2.77,3.14)$ & 1650 & $3.49(3.32,3.67)$ \\
\hline$\geq 35,000$ & 1022 & $3.89(3.65,4.14)$ & 1229 & $3.87(3.65,4.09)$ \\
\hline
\end{tabular}

a 5 year age group standardised to the whole CKB population

${ }^{\mathrm{b}}$ Crude incidence

No., number

A previous systematic review reported inconsistent associations of educational level and income with prevalent type 2

a

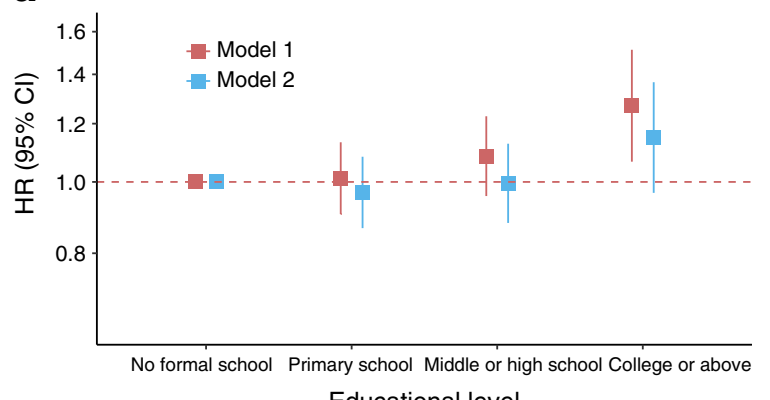

Educational level

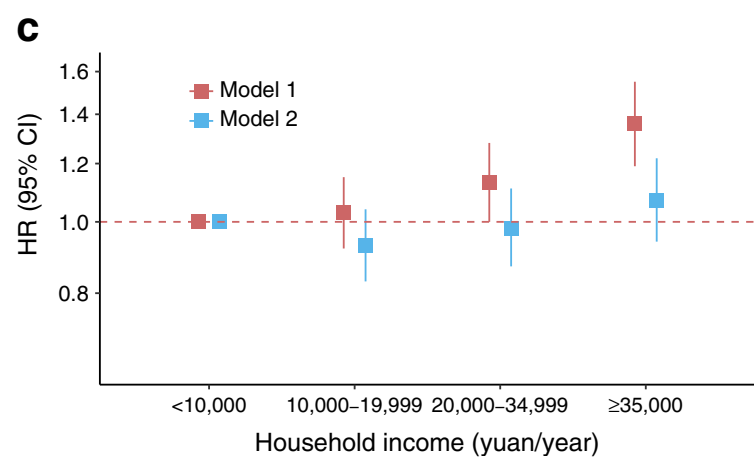

Fig. 2 Adjusted HRs and 95\% CIs for incident diabetes associated with educational level $(\mathbf{a}, \mathbf{b})$ and household income $(\mathbf{c}, \mathbf{d})$ in men $(\mathbf{a}, \mathbf{c})$ and women $(\mathbf{b}, \mathbf{d})$, plotted on a logarithmic scale. For educational level, model 1 was stratified by age at baseline ( 5 year age group) and study region, and adjusted for age at baseline (continuous), family history of diabetes and household income. Model 2 was further adjusted for BMI based on diabetes in China [7]. Most of the studies included in the systematic review had small sample sizes, were restricted to

b

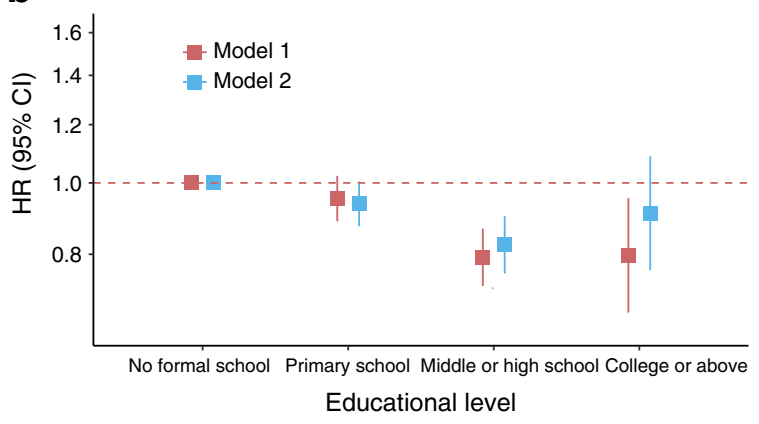

d

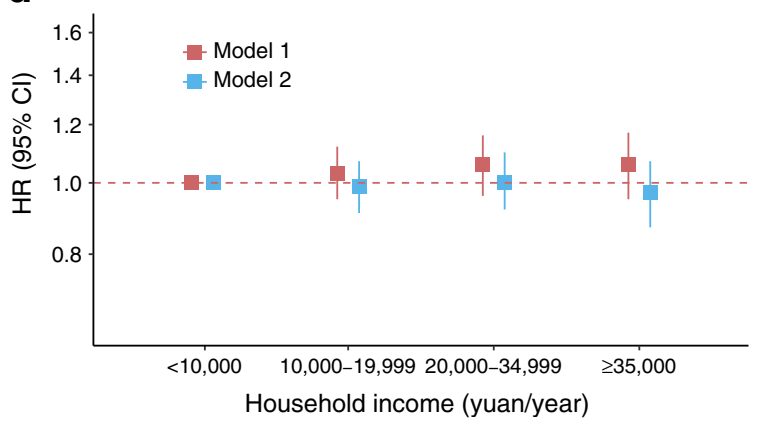

model 1 . For household income, model 1 was stratified by age at baseline (5 year age group) and study region, and adjusted for age at baseline (continuous), family history of diabetes, educational level and household size. Model 2 was further adjusted for BMI based on model 1. See ESM Tables 8 and 9 for data 
a particular geographic area and the association between SES and diabetes prevalence was rarely the primary research question. Few previous studies explored the association separately in men and women.

To our knowledge, our study is the first nationwide prospective study to describe the association between SES and diabetes incidence in China. The positive association between SES and incident diabetes in men in our study contrasts with findings from high-income countries [15] and Taiwan [16], but they are consistent with those from Thailand [17]. Evidence for an association between SES and incident diabetes from other LMIC is sparse [15]. A study of 10,704 Chinese adults living in Qingdao, a coastal city in China, reported an inverse association between educational level and incident diabetes in both sexes during 2001-11 [18]. In that study, a high proportion of both men and women had college education or higher (16.8\% and $24.0 \%$, respectively) as opposed to $7.9 \%$ and $4.5 \%$ in the present study and $8.7 \%$ of men and $6.1 \%$ of women in similar age groups in the 2010 Chinese census [19]. It is possible that the Qingdao findings represent the patterns of association in Chinese populations at a more advanced stage of epidemiological transition than other parts of China. The proportions of people in the highest education category in our study were similar to those of participants in the 2010 Chinese census. However, it is important to recognise that the patterns we observed may not reflect those in the whole population of China; the CKB sample was never intended to be nationally representative and the response rate was approximately $30 \%$ (comparing favourably with other large nationwide biobank studies, such as the UK Biobank).

SES has profound effects on health through complex processes, such as access to healthcare, health behaviours and environmental exposures [20]. The mechanisms through which SES influences the development of diabetes are not fully understood. The association between SES and diabetes may be partially explained by the distribution of conventional risk factors for diabetes such as being overweight or obese, which are strongly patterned by SES in many populations [13, 14]. In this study, the associations between SES and both diabetes prevalence and incidence were attenuated after adjustment for BMI, suggesting that BMI was likely to be a key mediator in the pathway linking SES and diabetes prevalence and incidence in the Chinese population. This finding was consistent with that from western countries [21].

We observed sex differences in the associations between educational level and diabetes prevalence and incidence. These sex differences can be at least partly attributed to differences in the relationship between education and BMI in men and women. Previous cross-sectional studies in China have reported that men with higher educational level were more likely to be overweight or obese than those of lower educational attainment, while the converse was true in Chinese women [22-24]. A review of studies from LMIC reported that the burden of obesity tends to shift from groups with high to low educational level during economic development, and this transition occurs earlier for women than men [25]. This evidence may explain the inconsistent findings between men and women in our study. Further exploration of the association between SES and BMI in China would be interesting but was beyond the scope of this study.

Interestingly, in women, we observed an inverse association between educational level and incident diabetes, while the association between household income and incident diabetes was positive (although not statistically significant). Compared with income, educational level is probably more strongly linked to an individual's cognitive functioning and healthpromoting behaviours, and plays a greater role in the onset of diseases, while income is more strongly related to the progression of diseases [26]. This suggests that educational level may be a more sensitive predictor of disease development than income during the epidemiological transition, at least in women. We found some evidence that the positive association between income and incident diabetes was weaker in both sexes in more economically developed geographic areas than in those at earlier stages of development. This may indicate that the transition in the association between SES and risk of diabetes from being positive to inverse is already occurring in more economically developed areas in China. However, more evidence is required to support this hypothesis.

Strengths and limitations A key strength of this study is that it is the first nationwide study to describe contemporary associations between SES and diabetes incidence in China. The large and diverse population sample permits the investigation of potential differences in the association between SES and diabetes in settings where economic development and epidemiological transition may be at different stages. Reviews of medical records in about 1000 incident cases identified a positive predictive value of $97 \%$ based on ADA diagnostic criteria [27] and medication use. In addition, extremely low loss to follow-up limits the potential for biased risk estimates.

One of the key study limitations is potential underascertainment of both prevalent and incident diabetes. The diabetes prevalence in the CKB was slightly lower than in contemporaneous nationally representative surveys in which diabetes diagnosis was based on a combination of self-report, fasting blood glucose, glycated haemoglobin measurement and oral glucose tolerance tests $[2,28]$. It is not clear whether under-ascertainment of prevalent diabetes differed across SES groups. For incident diabetes, only hospitalised diabetes events were identified in the CKB. Hospital admission rates may be greatly affected by people's ability to pay and vary by SES in China. If Chinese people of higher SES are more likely 
to be admitted to hospital, as reported by a previous study [29], then under-ascertainment of diabetes might be higher in lower SES groups; this would bias the association between SES and incident diabetes. We established that the proportion of prevalent diabetes that was self-reported increased with higher SES in both men and women in the CKB population. Our findings would therefore potentially have exaggerated the positive association between SES and incident diabetes in men, and the inverse association with educational level in women is likely to have been underestimated. Future research is required with more complete diabetes case ascertainment, such as from repeated cross-sectional measures of diabetes status using reliable laboratory tests. Furthermore, the statistical power of detecting a $6 \%$ relative difference in hazard of incident diabetes between the highest and lowest household income in women is low (power $=0.31$ ), which may contribute to the non-significant positive association between household income and incident diabetes. We were not able to identify type of diabetes, but most diabetes cases were likely to be type 2 diabetes given the age of the study population.

In summary, we found that among Chinese adults in mainland China, educational level was positively associated with prevalent and incident diabetes in men, but inversely associated with diabetes in women. Household income was positively associated with prevalent and incident diabetes in both sexes. The findings of this study provide useful information for identifying priority groups for prevention of diabetes and to allow evaluation of the effect of policies that influence health inequalities in China. Health and social policies that aim to reduce socioeconomic and geographic inequalities in diabetes burden should give priority to primary and secondary prevention of overweight and obesity in Chinese adults and take account of potentially different associations with SES in men and women. Future research is needed to identify effective approaches to reducing inequalities by SES in diabetes incidence and to investigate whether SES also affects the risk of developing diabetic complications in China.

Acknowledgements The chief acknowledgement is to the participants, the project staff and the China National Centre for Disease Control and Prevention (CDC) and its regional offices for access to death and disease registries. The Chinese National Health Insurance scheme provides electronic linkage to all hospital admission data.

Data availability Details of how to access the CKB data and details of the data release schedule are available from www.ckbiobank.org/site/Data+ Access.

Funding The baseline survey and the first re-survey were supported by a research grant from the Kadoorie Charitable Foundation in Hong Kong. The long-term continuation of the project is supported by programme grants from the UK Wellcome Trust (088158/Z/09/Z, 104085/Z/14/Z), the Chinese Ministry of Science and Technology (2011BAI09B01, 201214), the Chinese National Natural Science Foundation (81390540, $81390541,81390544)$ and the National Key Research and Development Program of China (2016YFC0900500,
2016YFC0900501, 2016YFC0900504, 2016YFC1303904). The British Heart Foundation (BHF), Medical Research Council and Cancer Research UK provide core funding to the Oxford Clinical Trial Service Unit. Hongjiang $\mathrm{Wu}$ acknowledges support from the China Scholarship Council. Fiona Bragg acknowledges support from the BHF Centre of Research Excellence, Oxford.

Duality of interest The authors declare that there is no duality of interest associated with this manuscript.

Contribution statement HW, FB, DG and SHW contributed to the concept and design of the study. HW and FB contributed to the data analysis. HW wrote the first draft of the paper. HW, FB, SHW and ZC had full access to all the data in the study and take responsibility for the integrity of the data and the accuracy of the analysis. All authors contributed to interpretation of data and revision of the paper and agreed to its publication.

Open Access This article is distributed under the terms of the Creative Commons Attribution 4.0 International License (http:// creativecommons.org/licenses/by/4.0/), which permits unrestricted use, distribution, and reproduction in any medium, provided you give appropriate credit to the original author(s) and the source, provide a link to the Creative Commons license, and indicate if changes were made.

\section{References}

1. Chan JC, Zhang Y, Ning G (2014) Diabetes in China: a societal solution for a personal challenge. Lancet Diabetes Endocrinol 2(12):969-979. https://doi.org/10.1016/S2213-8587(14)70144-5

2. Wang L, Gao P, Zhang M et al (2017) Prevalence and ethnic pattern of diabetes and prediabetes in China in 2013. JAMA 317(24): 2515-2523. https://doi.org/10.1001/jama.2017.7596

3. Ma RC (2018) Epidemiology of diabetes and diabetic complications in China. Diabetologia 61(6):1249-1260. https://doi.org/10. 1007/s00125-018-4557-7

4. Gong P, Liang S, Carlton EJ et al (2012) Urbanisation and health in China. Lancet 379(9818):843-852. https://doi.org/10.1016/S01406736(11)61878-3

5. World Health Organization (2010) Noncommunicable disease risk factors and socioeconomic inequalities - what are the links? A multicountry analysis of noncommunicable disease surveillance data. Available from https://apps.who.int/iris/handle/10665/207601? locale-attribute=en\&. Accessed 24 April 2019

6. Allen L, Williams J, Townsend N et al (2017) Socioeconomic status and non-communicable disease behavioural risk factors in lowincome and lower-middle-income countries: a systematic review. Lancet Glob Health 5(3):e277-e289. https://doi.org/10.1016/ S2214-109X(17)30058-X

7. Wu H, Meng X, Wild SH, Gasevic D, Jackson CA (2017) Socioeconomic status and prevalence of type 2 diabetes in mainland China, Hong Kong and Taiwan: a systematic review. J Glob Health 7(1):011103. https://doi.org/10.7189/ jogh.07.011103

8. Wu H, Jackson CA, Wild SH, Jian W, Dong J, Gasevic D (2018) Socioeconomic status and self-reported, screen-detected and total diabetes prevalence in Chinese men and women in 2011-2012: a nationwide cross-sectional study. J Glob Health 8(2):020501020501. https://doi.org/10.7189/jogh.08.020501

9. Chen Z, Chen J, Collins R et al (2011) China Kadoorie Biobank of 0.5 million people: survey methods, baseline characteristics and long-term follow-up. Int J Epidemiol 40(6):1652-1666. https:// doi.org/10.1093/ije/dyr120 
10. Du H, Bennett D, Li L et al (2013) Physical activity and sedentary leisure time and their associations with BMI, waist circumference, and percentage body fat in 0.5 million adults: the China Kadoorie Biobank study. Am J Clin Nutr 97(3):487-496. https://doi.org/10. 3945/ajen.112.046854

11. National Bureau of Statistics of China (2016) China statistical yearbook 2016, China. Available from www.stats.gov.cn/tjsj/ndsj/2016/ indexeh.htm. Accessed 24 April 2019

12. Zhou B (2002) Predictive values of body mass index and waist circumference for risk factors of certain related diseases in Chinese adults - study on optimal cut-off points of body mass index and waist circumference in Chinese adults. Biomed Environ Sci 15(1):83-96

13. Williams E, Tapp R, Magliano D, Shaw J, Zimmet P, Oldenburg B (2010) Health behaviours, socioeconomic status and diabetes incidence: the Australian Diabetes Obesity and Lifestyle Study (AusDiab). Diabetologia 53(12):2538-2545. https://doi.org/10. 1007/s00125-010-1888-4

14. Robbins JM, Vaccarino V, Zhang H, Kasl SV (2001) Socioeconomic status and type 2 diabetes in African American and non-Hispanic white women and men: evidence from the Third National Health and Nutrition Examination Survey. Am J Public Health 91(1):76-83

15. Agardh E, Allebeck P, Hallqvist J, Moradi T, Sidorchuk A (2011) Type 2 diabetes incidence and socio-economic position: a systematic review and meta-analysis. Int J Epidemiol 40(3):804-818. https://doi.org/10.1093/ije/dyr029

16. Hsu C-C, Lee C-H, Wahlqvist ML et al (2012) Poverty increases type 2 diabetes incidence and inequality of care despite universal health coverage. Diabetes Care 35(11):2286-2292. https://doi.org/ $10.2337 / \mathrm{dc} 11-2052$

17. Papier K, Jordan S, Catherine DE et al (2016) Incidence and risk factors for type 2 diabetes mellitus in transitional Thailand: results from the Thai cohort study. BMJ Open 6(12):e014102. https://doi. org/10.1136/bmjopen-2016-014102

18. Shang X, Li J, Tao Q et al (2013) Educational level, obesity and incidence of diabetes among Chinese adult men and women aged 18-59 years old: an 11-year follow-up study. PLoS One 8(6): e66479. https://doi.org/10.1371/journal.pone.0066479

19. National Bureau of Statistics of China (2011) 2010 population census of people's republic of China. Available from www.stats.gov.cn/ english/Statisticaldata/CensusData/rkpc2010/indexch.htm. Accessed 17 April 2018
20. Adler NE, Newman K (2002) Socioeconomic disparities in health: pathways and policies. Health Aff 21(2):60-76. https://doi.org/10. 1377/hlthaff.21.2.60

21. Espelt A, Borrell C, Palència L et al (2013) Socioeconomic inequalities in the incidence and prevalence of type 2 diabetes mellitus in Europe. Gac Sanit 27(6):494-501. https://doi.org/10.1016/j.gaceta. 2013.03.002

22. Pei L, Cheng Y, Kang Y, Yuan S, Yan H (2015) Association of obesity with socioeconomic status among adults of ages 18 to 80 years in rural Northwest China. BMC Public Health 15(1):160. https://doi.org/10.1186/s12889-015-1503-1

23. Zhang H, Xu H, Song F, Xu W, Pallard-Borg S, Qi X (2017) Relation of socioeconomic status to overweight and obesity: a large population-based study of Chinese adults. Ann Hum Biol 44(6): 495-501. https://doi.org/10.1080/03014460.2017.1328072

24. Jones-Smith JC, Gordon-Larsen P, Siddiqi A, Popkin BM (2012) Emerging disparities in overweight by educational attainment in Chinese adults (1989-2006). Int J Obes 36(6):866-875. https:// doi.org/10.1038/ijo.2011.134

25. Monteiro CA, Moura EC, Conde WL, Popkin BM (2004) Socioeconomic status and obesity in adult populations of developing countries: a review. Bull World Health Organ 82(12):940-946

26. Herd P, Goesling B, House JS (2007) Socioeconomic position and health: the differential effects of education versus income on the onset versus progression of health problems. J Health Soc Behav 48(3):223-238. https://doi.org/10.1177/002214650704800302

27. American Diabetes Association (2017) 2. Classification and diagnosis of diabetes. Diabetes Care 40(Supplement 1):S11-S24. https://doi.org/10.2337/dc17-S005

28. Xu Y, Wang L, He J et al (2013) Prevalence and control of diabetes in Chinese adults. JAMA 310(9):948-959. https://doi.org/10.1001/ jama.2013.168118

29. Wang HH, Wang JJ, Lawson KD et al (2015) Relationships of multimorbidity and income with hospital admissions in 3 health care systems. Ann Fam Med 13(2):164-167. https://doi.org/10. 1370/afm. 1757

Publisher's note Springer Nature remains neutral with regard to jurisdictional claims in published maps and institutional affiliations. 